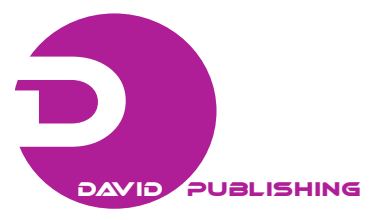

\title{
Evaluation of Dynamic Behavior of Bedrock Foundation Based on FEM and DEM Simulations
}

\author{
Yujing Jiang \\ Department of Civil Engineering, Faculty of Engineering, Nagasaki University, Nagasaki 852-8521, Japan
}

\begin{abstract}
The seismic behavior of the bedrock foundation during earthquakes concerns the stability and safety of nuclear power plants. Discontinuities like joints and faults existing in rock masses affect significantly the dynamic behavior of bedrock. The dynamic FEM (finite element method) has been commonly utilized to analyze the seismic responses of bedrock, however, it cannot well represent the large deformation behavior of discontinuities. The DEM (distinct element method) has a better capability of simulating the sliding and separation of discontinuities existing in the bedrock, which influence the propagation of seismic waves. In this study, the dynamic FEM and DEM simulations were carried out to investigate the seismic behavior of the bedrock foundation under a nuclear power plant, and the differences between those two methods were illuminated. Numerical simulation results indicate that the FEM underestimates the attenuation effect of faults on the propagation of seismic waves. With the capability of simulating large deformation behavior of discontinuities, the DEM can be regarded as a better method for studying the seismic responses of bedrock foundation which contains discontinuities.
\end{abstract}

Key words: Seismic behavior, numerical simulation, rock foundation, discontinuity, FEM \& DEM.

\section{Introduction}

As an efficient and clean energy, the nuclear energy has been developed and adopted by an increasing number of countries to secure their energy supplies and to reach their future objective of sustainability [1]. During the development of the nuclear power industry, the seismic resistance performance of nuclear power plants has always been one of the most crucial issues, concerning the safety of nuclear power plants. At present, most of the nuclear power plants have been built on bedrock foundations to minimize seismic shaking during earthquakes. Therefore, the dynamic behavior of bedrock foundation subjected to seismic loads affects the stability and safety of nuclear plants to a great extent. Moreover, there are a mass of discontinuities like joints and faults existing in the bedrock, with different sizes and shapes, weakening the mechanical properties of the bedrock [2]. The

Corresponding author: Yujing Jiang, Dr., Eng., professor, research fields: rock mechanics, stability of foundation and slope, geotechnical and geoenvironmental engineering, natural disaster science. E-mail: jiang@nagasaki-u.ac.jp. influence of discontinuities on the seismic behavior of bedrock remains as a fundamental and promising problem in the academic and engineering aspects.

The research on the seismic responses of foundations initiated from the simplified 1-D model of uniform horizontal soil layers with seismic waves propagating vertically to the ground surface. Schnabel et al. developed the code of SHAKE to investigate the seismic responses of viscoelastic layered soil [3]. Seed et al. proposed the relationship between the shear stiffness and damping coefficient and the strain for clay and sand materials, and gave suggestions for non-linear dynamic calculations [4]. Through the FEM (finite element method), Idriss et al. carried out numerical studies on the seismic responses of foundation, taking into account the variable damping, the results of which corresponded well with the field data [5]. In recent years, more and more efforts have been put into the studies on the seismic response of foundations due to its increasing importance. Kamiyama \& Yanagisawa performed researches on the influences of some factors, including the hypocenter characteristics, the 
propagation path of seismic waves and the mechanical properties of sites, on the seismic behavior of ground foundation [6]. Ushiyama et al. investigated the seismic characteristics of the Rokko fault rock located in Kansai, Japan by using FEM simulations [7]. Zhao et al. established a coupling model of finite and infinite elements to investigate the influences of the properties of soil and seismic waves on seismic responses [8]. Chen et al. developed an FEM code of SR2D to investigate the seismic responses of soil foundation, taking into account the effects of artificial boundary conditions, model dimensions and the damping coefficients of media [9].

Although tremendous efforts have been made, the seismic behavior of bedrock foundations is still not well understood, especially in the cases with involvement of discontinuities. The FEM has been commonly adopted in most of the previous studies, however, as a method of continuum mechanics, the FEM cannot represent well the large deformation behavior of discontinuities contained in rock masses, which is an important factor to affect the propagation of seismic waves. The DEM (distinct element method) attracts more and more attentions after it was proposed, due to its remarkable advantages in aspects of the non-linear dynamic calculation as well as the simulation of discontinuities' large deformations like sliding and separation [10]. In this study, both FEM and DEM were adopted to investigate the seismic responses of the bedrock foundation under a nuclear power plant located in Japan. Through the comparison of numerical results, the differences between those two methods in seismic simulations ware illuminated.

\section{Characteristics of Bedrock and Seismic Wave}

The bedrock of the objective nuclear plant located in Japan belongs to Mesozoic cumulates. The results of geophysical prospecting reveal that there are three major faults existing in this bedrock, with a controlling influence on the mechanical behavior of this bedrock.
The dip angles of these faults are $72^{\circ}, 56^{\circ}$ and $63^{\circ}$ respectively. The physical-mechanical properties of the intact rock and fractures were estimated through lab tests on rock samples taken from the objective ground, with the test results shown in Table 1. It can be found that the intact rock of this site has high strength, therefore, in this case, the macro mechanical behavior of this bedrock is principally affected by the existing rock faults.

Comparing to artificial waves, the real seismic waves taken from similar sites will be more representative to the situation when earthquake happens. In this study, the seismic acceleration waves measured at the Ojiya observation site during the M6.8 Chuetsu offshore earthquake (Niigata, July 16th, 2007) were adopted. As shown in Fig. 1, this seismic record consists of three components, which represent the seismic motions at the NS, EW and UD directions respectively. Due to the great destructiveness of shear wave to the rock masses and buildings, the EW component (with larger amplitude than the NS component) was selected as the input seismic wave in numerical simulations.

\section{Simulation of Seismic Wave at the Deep Rock Formation}

The seismic records obtained from the observation sites represent the seismic motions of the ground surface. During earthquakes, seismic waves propagate

Table 1 Physico-mechanical properties of the intact rock and per-existing fractures.

\begin{tabular}{lllll}
\hline & Parameter & Index & Unit & Value \\
\hline \multirow{4}{*}{ Intact } & Density & $\rho$ & $\mathrm{g} / \mathrm{cm}^{3}$ & 2.66 \\
rock & Elastic modulus & $E$ & $\mathrm{GPa}$ & 71 \\
& Poisson's ratio & $v$ & - & 0.18 \\
& Tensile strength & $\sigma_{t}$ & $\mathrm{MPa}$ & 10.2 \\
& Cohesion & $c_{r}$ & $\mathrm{MPa}$ & 22.3 \\
& Friction angle & $\varphi_{r}$ & $\circ$ & 62 \\
\hline \multirow{4}{*}{ Fracture } & Normal stiffness & $K_{n}$ & $\mathrm{MPa} / \mathrm{m}$ & 31.78 \\
& Shear stiffness & $K_{s}$ & $\mathrm{MPa} / \mathrm{m}$ & 3.22 \\
& Cohesion & $c_{f}$ & $\mathrm{MPa}$ & 0.027 \\
& Friction angle & $\varphi_{f}$ & $\circ$ & 35.9 \\
\hline
\end{tabular}




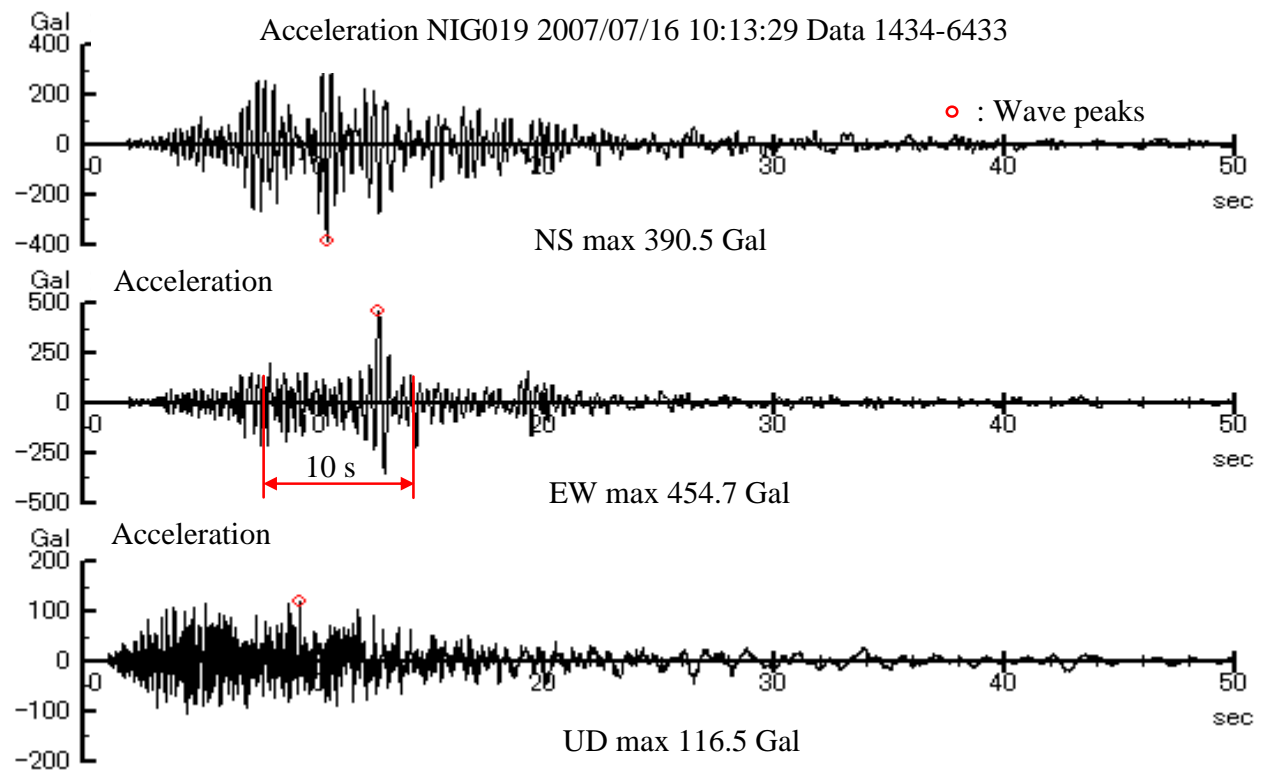

Fig. 1 Acceleration waves obtained at the Ojiya observation site during the Niigata earthquake.

from the deep rock formation to the ground surface. To reproduce such a process in numerical simulations, it is necessary to investigate the seismic motion at the deep rock formation firstly.

The code of $k$-SHAKE based on the repeated reflection theory of seismic waves, which is developed by the Structure and Planning Institute of Japan [11], was used to investigate the seismic motion at the deep rock formation under the proposed nuclear power plant with a depth of $200 \mathrm{~m}$. Comparing with the height of the nuclear buildings (the reactor building and the turbine building), the bedrock with a depth of $200 \mathrm{~m}$ is enough to represent the seismic behavior of the bedrock and the buildings. According to the results of in-situ tests, the propagation velocity of S-wave and the damping ratio were set as $3,363 \mathrm{~m} / \mathrm{s}$ and $3 \%$ respectively during the calculation.

Due to the time-consuming problem in dynamic calculations, only a part of the seismic wave at the deep rock formation, which lasts for 10 seconds (6.8-10.8 s in Fig. 1) and contains the maximum amplitudes of the total wave, was selected as the input wave in the numerical simulations. According to the different calculation principles of FEM and DEM, the velocity wave and acceleration wave were input to the DEM and FEM models respectively (Fig. 2).

\section{Numerical Simulations Based on FEM and DEM}

Numerical simulations of the seismic behavior of bedrock foundation under the nuclear power plant were carried out by using the FEM code of SuperFLUSH/2D [12] and the DEM code of UDEC [13]. Through comparing the results obtained from FEM and DEM simulations, the differences between those two methods in seismic response simulations were investigated.

\subsection{Numerical Models of FEM and DEM}

According to the dimensions of the nuclear buildings, the model size of the bedrock foundation was determined as $200 \mathrm{~m} \times 660 \mathrm{~m}$. Three major faults existing in the bedrock mentioned above were considered during simulations. The FEM and DEM models were established with the same sizes and rock structures (Fig. 3). To investigate the seismic responses at different locations with varying depths, four monitoring points were set in the middles of the FEM and DEM models, marked as points A, B, C and D 


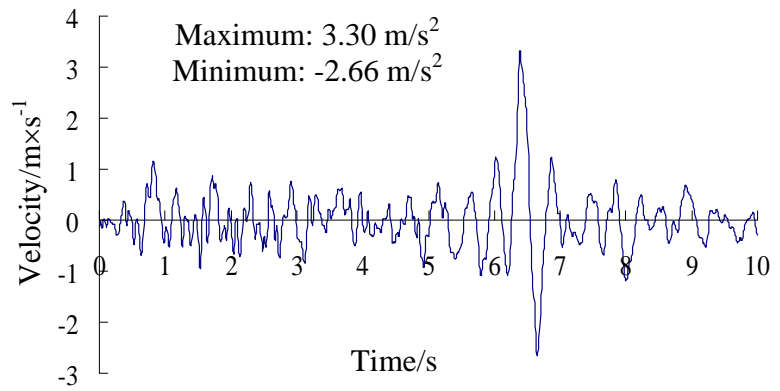

(a) Acceleration wave for FEM simulation

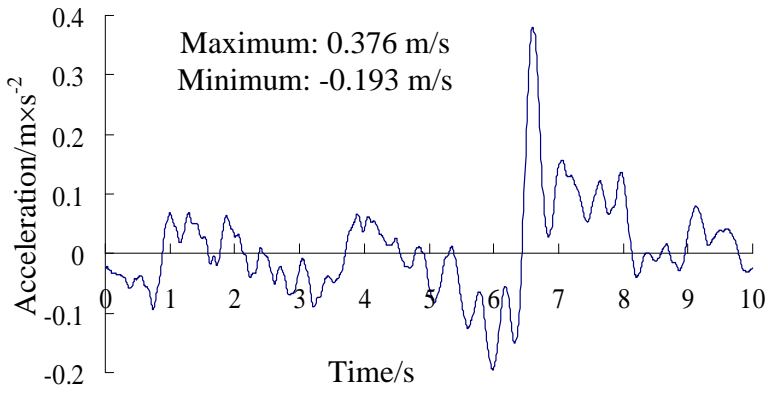

(b) Velocity wave for DEM simulation

Fig. 2 Seismic waves (6.8-16.8 s) at the deep rock formation with a depth of $200 \mathrm{~m}$.

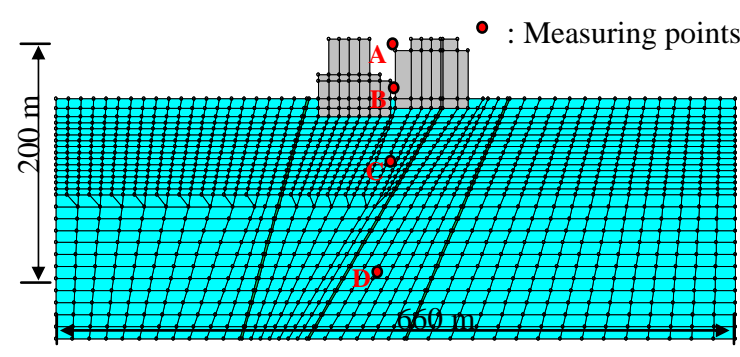

(a) FEM model

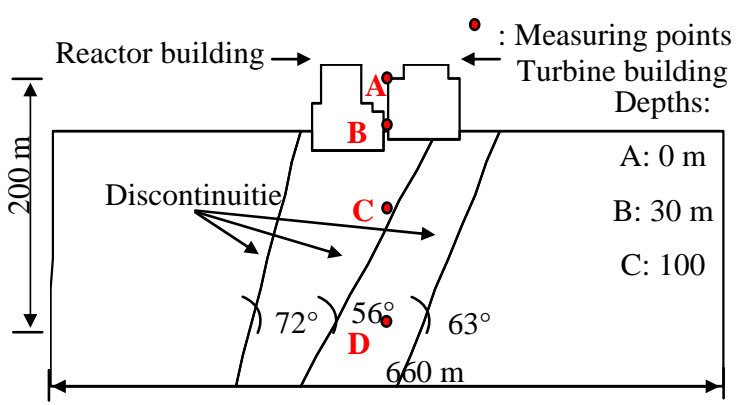

(b) DEM model

Fig. 3 Numerical models of FEM and DEM.

with the depths of $0 \mathrm{~m}, 30 \mathrm{~m}, 100 \mathrm{~m}$ and $190 \mathrm{~m}$ respectively.

In the FEM model, the plane strain elements and joint elements were used to represent the bedrock and faults respectively. In the DEM model, the Mohr-coulomb elements and Coulomb joint elements were used for the bedrock and faults. Triangular zones with a side length of $10 \mathrm{~m}$ were generated in the blocks of DEM model to calculate the internal deformations.

The upward-propagating seismic wave was input from the bottoms of numerical models. In both the DEM and FEM models, the viscous boundaries were assigned to the model bottoms to absorb the wave energy dissipating from the model interior. Lateral boundaries of models were set as free semi-infinite regions (the free-field boundaries), ensuring the propagation of seismic waves out of the model.

\subsection{Numerical Results}

At different locations, the response waves of the bedrock foundation differ due to the influences of rock properties and the rock structure. Fig. 4 shows the horizontal velocity waves at four monitoring points with varying depths obtained from the FEM and DEM simulations. The FEM results (Fig. 4a) indicate that despite of the difference in depths, the response waves at different monitoring points exhibit a good consistency in the waveform, mainly in the aspects of the frequency and the time when seismic waves reach their peaks and troughs. Due to the fact that seismic waves need only about $0.06 \mathrm{~s}$ to travel through the bedrock with a depth of $200 \mathrm{~m}$, the time corresponding to the peaks and troughs of waves at different locations agrees well with each other, and no obvious delay phenomenon was observed. On the other hand, the depth has an obvious influence on the amplitudes at peaks and troughs of the waves. Fig. 4 a reveals that during the propagation process of seismic waves from the deep rock formation to the ground surface, the amplitudes at wave's peaks and troughs increase. It can be found that the hard bedrock has an amplification effect on the amplitudes of the seismic waves in the FEM simulation. 


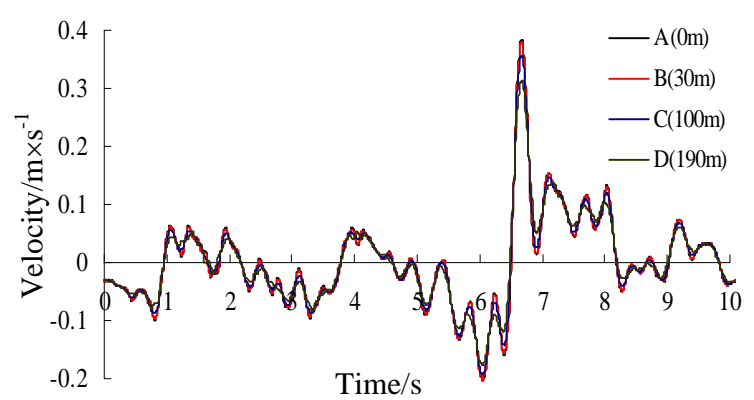

(a) FEM results

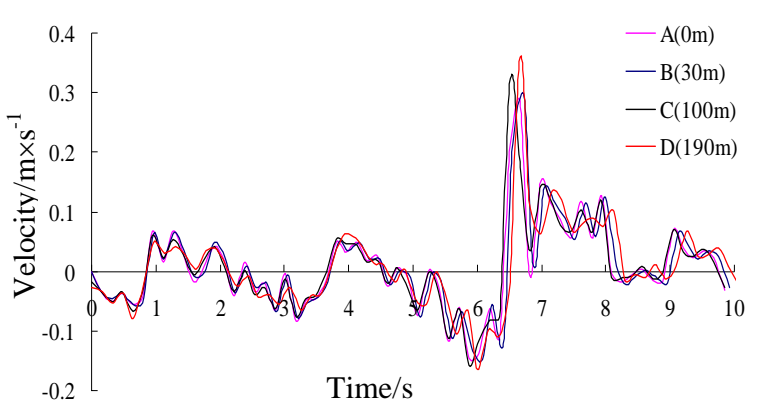

(b) DEM results

Fig. 4 Horizontal velocity waves at different monitoring points in the FEM and DEM models.

For the DEM results, the velocity waves at different monitoring points have similar waveforms comparing with the input waveform (Fig. 4b). However, the response waves obtained through the DEM simulation show different characteristics from the FEM results, mainly in two aspects: the amplitude variation at peaks and troughs of waves is more obvious, the time corresponding to wave peaks and troughs exhibits deviation to some extent. Due to the existence of faults, the response waves have different waveforms at different monitoring points. With the upward propagation of seismic waves, the amplitudes of response waves decrease. In addition, the time deviation at the peaks and troughs of waves becomes obvious as waves propagate across faults.

\subsection{Comparison of FEM and DEM Results}

The numerical results of FEM and DEM present different characteristics, mainly in the aspects of waveform divergence and depth's effect on wave amplitudes. It can be speculated that those differences are resulted from the different treatments of discontinuities in FEM and DEM simulations. The existing discontinuities have an attenuation effect on the propagation of seismic waves, which has been reported in a number of literatures [14-16]. When seismic waves propagate across discontinuities, the scattering of waves occurs, such as reflection, refraction, transmission and diffraction, resulting in the dissipation of seismic energy. Therefore, the spatial distribution and deformation behavior of discontinuities affect the propagation characteristics of seismic waves in the bedrock to a great extent.

In the FEM model, although the physicalmechanical parameters of faults were assigned, the fault elements were still connected to the rock elements, and the stresses and displacements across the faults were continuous. The faults in the FEM model can be regarded as welded interfaces, which cannot simulate the large deformation behavior of faults, such as sliding and separation. The FEM underestimates the fault's attenuation effect on the propagation of seismic waves. Therefore, the response waves at different locations show a good consistency, and the hard bedrock has an amplification effect on the wave amplitudes.

As a different method, the DEM treats the faults as displacement discontinuities (non-welded interfaces), and can simulate the fault's large deformation behavior, with a much better ability to present the effect of discontinuities. Within the DEM simulations, due to that the deformation behavior of faults in bedrock cause the scatting of seismic waves and the dissipation of seismic energy, the wave amplitude decreases during the wave propagation. In addition, the faults also result in divergence of waveforms at different locations.

To make a further comparison of the numerical results obtained from FEM and DEM simulations, the response waves at the same monitoring points were analyzed (Fig. 5). Those results indicate that the amplitudes of response waves obtained from DEM simulations are smaller than those of waves obtained 


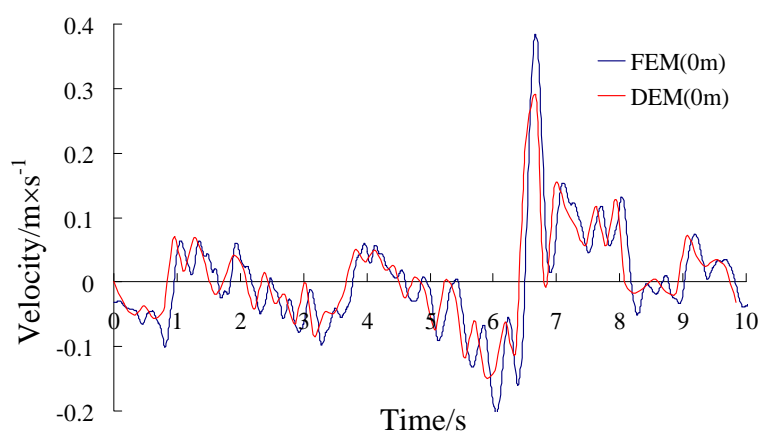

(a) Depth: $0 \mathrm{~m}$

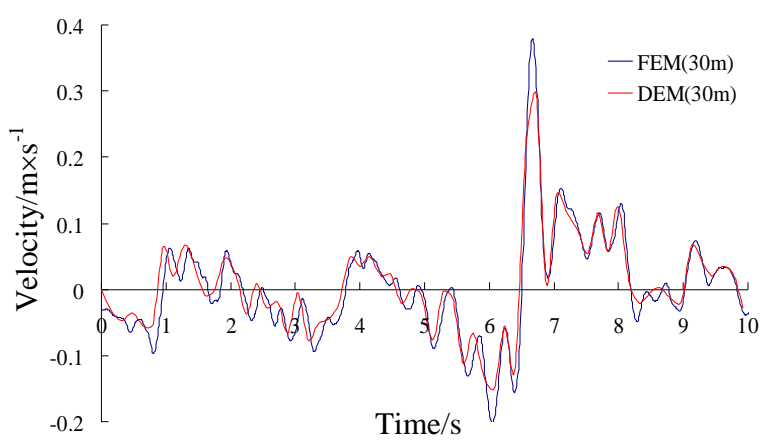

(b) Depth: $30 \mathrm{~m}$

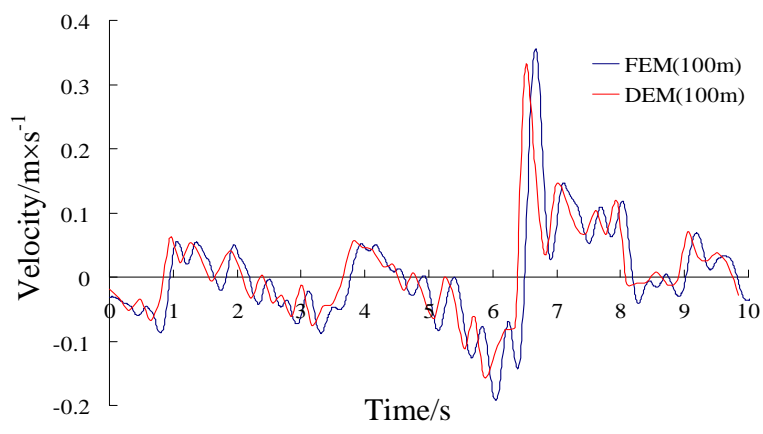

(c) Depth: $100 \mathrm{~m}$

Fig. 5 Comparison of response waves at different locations obtained from FEM and DEM simulations.

from FEM simulations, due to that the deformations of pre-existing faults in the DEM model cause energy dissipation and perform an attenuation effect during the propagation of seismic waves. In addition, the difference in wave amplitudes becomes more obvious when the depths of monitoring points decrease.

\section{Conclusions and Discussions}

The seismic behavior of the bedrock foundation concerns the stability and safety of nuclear plants. In this study, both the FEM and DEM were adopted to investigate the seismic response of the bedrock foundation under a nuclear power plant located in Japan. The difference between those two methods in seismic simulations was investigated.

The EW component of the seismic wave obtained from the Ojiya observation site during the M6.8 Chuetsu offshore earthquake (Niigata, July 16th, 2007) was adopted in this study. The code of $k$-SHAKE was used to investigate the seismic motion at the deep rock formation with a depth of $200 \mathrm{~m}$. The corresponding velocity and acceleration waves were input into the DEM and FEM models, respectively.

The response waves at different locations obtained from FEM simulations exhibit a good consistency. With seismic waves propagating from the deep rock formation to the ground surface, the amplitudes at waves' peaks and troughs increase. However, according to the DEM results, the amplitudes of seismic waves decrease with the upward propagation of seismic waves. In addition, the DEM simulations show obvious divergences of waveforms. The comparison of response waves at the same locations indicates that the wave amplitudes obtained from DEM simulations are smaller than those of the waves obtained from FEM simulations. Moreover, the difference in wave amplitudes becomes obvious with the decrease of depths of monitoring points.

The differences between the FEM and DEM simulations were resulted from the different treatments of discontinuities. The FEM cannot present well the large deformation behavior of faults, underestimating the fault's attenuation effect on the propagation of seismic waves. Relatively, the DEM, with a better capability of simulating the sliding and separation of faults, can evaluate the effect of discontinuities well in seismic simulations. Therefore, the DEM can be regarded as a better method than the FEM in evaluations of seismic response of bedrock containing discontinuities. The main results in this paper may provide suggestions for numerical studies and safety evaluations of bedrock subjected to seismic loads. 


\section{Acknowledgments}

This work was financially supported by the PCSIRT (the Program for Changjiang Scholars and Innovative Research Team in University) and the National Science Fund for Distinguished Young Scholars (No. 50028403) of P. R. China

\section{References}

[1] K. Fiore, Nuclear energy and sustainability: Understanding ITER, Energy Policy 34 (2006) 3334-3341.

[2] Y. Jiang, B. Li and Y. Yamashita, Simulation of cracking near a large underground cavern in a discontinuous rock mass using the expanded distinct element method, International Journal of Rock Mechanics and Mining Sciences 46 (2009) 97-106.

[3] P. B. Schnabel, J. Lysmer and H. B. Seed, SHAKE: A computer program for earthquake response analysis of horizontally layered sites, EERC University of California, Berkeley, 1972.

[4] H. B. Seed, R. T. Wong, I. M. Idriss and K. Tokimatsu, Moduli and damping factors for dynamic analyses of cohesionless soils, Journal of Geotechnical Engineering 112 (11) (1986) 1016-1032.

[5] I. M. Idriss, H. B. Seed and N. Serff, Seismic response by variable damping finite elements, Journal of Geotechnical Engineering Division 100 (1) (1974) 1-13.

[6] M. Kamiyama and E. Yanagisawa, A statistical model for estimating response spectra of strong earthquake ground motions with emphasis on local soil condition, Soils and Foundations 26 (2) (1986) 16-32.

[7] N. Ushiyama, H. Taniyama and F. Tsunoda, Concussional movement of the Rokko fault block in
Kansai and its dynamic response analysis for seismic isolation block system, Earth Science 26 (2009) 343-354.

[8] C. Zhao, C. Zhang, G. Wang and G. Zhang, Effects of the characteristics of soil lays on field seismic response by using infinite element, Hydro-Science and Engineering 4 (1988) 31-45.

[9] C. Chen, M. Lou and S. Tao, Discussions on two-dimensional finite element analysis of seismic response, Technology for Earthquake Disaster Prevention 1 (4) (2006) 292-301.

[10] C. Zhang, O. A. Pekau, F. Jin and G. Wang, Application of distinct element method in dynamic analysis of high rock slopes and blocky structure, Soil Dynamics and Seismic Engineering 116 (1997) 385-394.

[11] Structure and Planning Institute of Japan, User manual of the seismic response analysis program for stratified soil layers: $k$-SHAKE, Tokyo, 2005.

[12] Structure and Planning Institute of Japan, User manual of the two-dimensional dynamic interaction analysis program for ground and structure: SuperFLUSH/2D, Tokyo, 2005.

[13] Itasca Consulting Group Inc., Universal Distinct Element Code: Theory and Background, Minnesota, 2004.

[14] J. G. Cai and J. Zhao, Effects of multiple parallel fractures on apparent attenuation of stress waves in rock masses, International Journal of Rock Mechanics and Mining Sciences 37 (2000) 661-682.

[15] J. Zhao and J. G. Cai, Transmission of elastic P-waves across single fractures with a nonlinear normal deformational behavior, Rock Mechanics and Rock Engineering 34 (1) (2001) 3-22.

[16] X. B. Zhao, J. Zhao, J. G. Cai and A. M. Hefny, UDEC modeling on wave propagation across fractured rock masses, Computers and Geotechnics 35 (2008) 97-104. 